Review

\title{
Modulation of purinergic signaling by NPP-type ectophosphodiesterases
}

\author{
Cristiana Stefan, Silvia Jansen \& Mathieu Bollen \\ Division of Biochemistry, Department of Molecular Cell Biology, Faculty of Medicine, KULeuven, \\ B-3000 Leuven, Belgium
}

Received 20 October 2005; accepted in revised form 11 November 2005; Published online 1 June 2006

Key words: alkaline sphingomyelinase, ectonucleotide pyrophosphatase, ectophosphodiesterase, ENPP, lysophospholipase D, NPP, NPP-type ectophosphodiesterase

\begin{abstract}
Extracellular nucleotides can elicit a wide array of cellular responses by binding to specific purinergic receptors. The level of ectonucleotides is dynamically controlled by their release from cells, synthesis by ectonucleoside diphosphokinases and ectoadenylate kinases, and hydrolysis by ectonucleotidases. One of the four structurally unrelated families of ectonucleotidases is represented by the NPP-type ectophosphodiesterases. Three of the seven members of the NPP family, namely NPP1-3, are known to hydrolyze nucleotides. The enzymatic action of NPP1-3 (in)directly results in the termination of nucleotide signaling, the salvage of nucleotides and/or the generation of new messengers like ADP, adenosine or pyrophosphate. NPP2 is unique in that it hydrolyzes both nucleotides and lysophospholipids and, thereby, generates products that could synergistically promote cell motility. We review here the enzymatic properties of NPPs and analyze current evidence that links their nucleotide-hydrolyzing capability to epithelial and neural functions, the immune response and cell motility.
\end{abstract}

Abbreviations: Ado-adenosine; ADP-adenosine 5' diphosphate; AMP-adenosine 5' monophosphate; $\mathrm{Ap}_{\mathrm{n}} \mathrm{A}$ - diadenosine polyphosphate; ATP - adenosine $5^{\prime}$ triphosphate; CSF - cerebrospinal fluid; E-NTPDases - ectonucleoside triphosphate diphosphohydrolases; GPC-glycerophosphorylcholine; LPA-lysophosphatidic acid; LPC-lysophosphatidyl choline; $\mathrm{MCC}$ - mucociliary clearing; $\mathrm{NAD}^{+}$- nicotinamide adenine dinucleotide; $\mathrm{NMN}$ - nicotinamide mononucleotide; NPP nucleotide pyrophosphatase/phosphodiesterase; $\mathrm{P}_{\mathrm{i}}$ - inorganic phosphate; $\mathrm{PP}_{\mathrm{i}}-$ pyrophosphate; $\mathrm{SM}$ - sphingomyelin; $\mathrm{SMB}$ somatomedin-B like domain; S-S bridges - disulfide bridges; UDP-glucose - uridine diphosphate glucose

\section{Introduction}

Extracellular nucleotides function as autocrine or paracrine signaling molecules. Their binding to P2 purinergic receptors leads to both short-term and long-term effects [1]. Short-term effects, like neurotransmission and hormone secretion, are mediated by the ionotropic P2X receptors that bind mainly ATP, while long-term effects, including cell proliferation, differentiation and migration, occur via the metabotropic G-protein coupled P2Y receptors that interact with a broader range of nucleotides [2-5]. In keeping with their importance in cell signaling, the extracellular concentration of nucleotides is tightly regulated. Nucleotides can be released from cells primarily by exocytosis or selective transport through the plasma membrane $[1,6]$, but they can also be generated extracellularly by nucleoside diphosphokinase and adenylate kinase activities [7-9] (Figure 1). There are four major

Correspondence to: Cristiana Stefan, Afdeling Biochemie, Campus Gasthuisberg, O\&N1, Herestraat 49, B-3000 Leuven, Belgium. Tel: +321-6345700; Fax: +32-1-6345995; E-mail: Christiana.Stefan@med.kuleuven.be families of ectonucleotidases, namely E-NTPDases (ectonucleoside triphosphate diphosphohydrolases), alkaline phosphatases, NPP-type ectophosphodiesterases and the $5^{\prime}$-nucleotidase [10]. Often these ectonucleotidases work in concert or consecutively (Figure 1). ATP, for instance, can be degraded in one step to either ADP or AMP by ENTPDase or NPP isoenzymes. ADP can be further hydrolyzed to AMP by E-NTPDases and NPPs, and AMP is converted to adenosine by alkaline phosphatases or the 5 -nucleotidase. This sequential degradation mechanism not only terminates ATP signaling but also generates intermediates with distinct signaling properties. Thus, ADP selectively interacts with P2 receptor subtypes and is the principal platelet recruiting factor $[11,12]$, while adenosine, acting through P1 receptors, plays a major role in the regulation of blood flow and the immune response [13-15]. The relative contribution of the distinct ectonucleotidase species to the modulation of purinergic signaling may depend on differential tissue and cell distribution, regulation of expression, targeting to specific membrane domains, but also on substrate availability and substrate preference. For example, the localization of NTPDase 1 and 


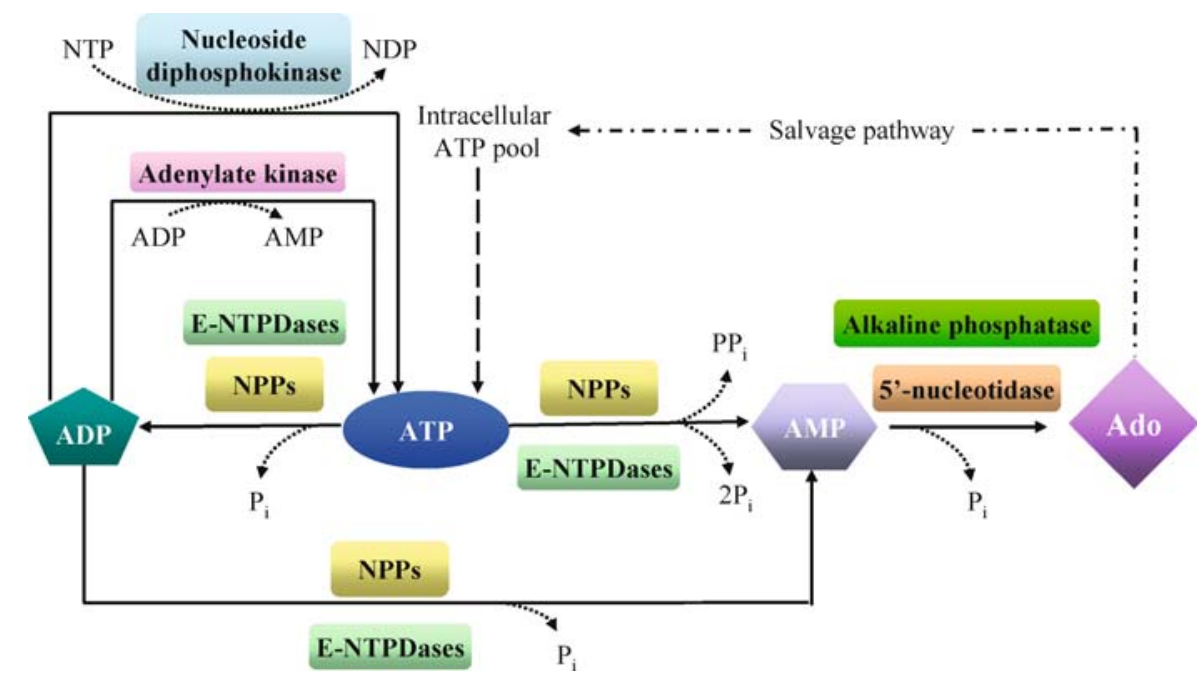

Figure 1. NPPs are part of the extracellular nucleotide-metabolizing network. The concentration of nucleotides in the extracellular milieu is the net result of the release of nucleotides from cells, their synthesis by nucleoside diphosphokinases and adenylate kinases, and their hydrolysis by ectonucleotidases. The examples shown here apply to ATP, the prototype extracellular nucleotide. Members of the E-NTDPase family, also known as apyrases, generally act as ATP diphosphohydrolases and hydrolyze ATP to ADP $+\mathrm{P}_{\mathrm{i}}$, and ADP to AMP $+\mathrm{P}_{\mathrm{i}}$, or ATP directly to AMP $+2 \mathrm{P}_{\mathrm{i}}$. Individual members however display substrate preference. For instance, E-NTPDase-1 metabolizes equally well ATP and ADP, while E-NTPDase-2 prefers ATP as a substrate. ATP can be regenerated from ADP by nucleoside diphosphokinase or adenylate kinase. NPPs, at least NPP1-3, have a nucleotide pyrophosphatase activity and metabolize ATP directly to ADP $+\mathrm{P}_{\mathrm{i}}$ or to AMP $+\mathrm{PP}_{\mathrm{i}}$. The hydrolysis of AMP to adenosine by $5^{\prime}$-nucleotidase/CD73 completes the dephosphorylation pathway of ATP. Adenosine can be taken up by cells such as lymphocytes and be re-used for intracellular nucleotide synthesis (nucleotide salvage). Several ectonucleotide species can be expressed by a given cell type, but the relative abundance, sorting to specific membrane domains and substrate availability are ultimately responsible for the net outcome of the nucleotide metabolism at the cell surface.

NTPDase 2 to distinct cell types within the vascular wall, combined with their substrate preference, may have direct implications for the control of platelet activation and coagulation responses in vivo $[16,17]$. The former ectonucleotidase metabolizes both ATP and ADP while the latter acts primarily as an ATP phosphohydrolase.

The family of NPPs (nucleotide pyrophosphatases/ phosphodiesterases) consists of seven members, numbered according to their order of cloning (Figure 2). Only NPP13 , which have a common ancestor, have been implicated in the hydrolysis of nucleotides [10, 18, 19], while NPP6-7 are only known to hydrolyze phosphodiester bonds in lysophospholipids or other choline phosphodiesters [20-22]. Remarkably, NPP2 acts on both nucleotides and lysophospholipids [23, 24]. Established nucleotide(-derived) substrates of NPP1-3 include ATP, diadenosine polyphosphates $\left(\mathrm{Ap}_{\mathrm{n}} \mathrm{A}\right)$, UDP-glucose, $\mathrm{NAD}^{+}$and $3^{\prime}-$ phosphoadenosine-5'-phosphosulfate (Figure 2). In vitro, NPP isoenzymes have an alkaline optimum $\mathrm{pH}$, but the physiological relevance of this property has not been explored.

Except for NPP2, all NPPs are single-span transmembrane proteins. NPP1 and NPP3 have a type-II orientation, with their N-terminus facing the cytosol, while NPP4-7

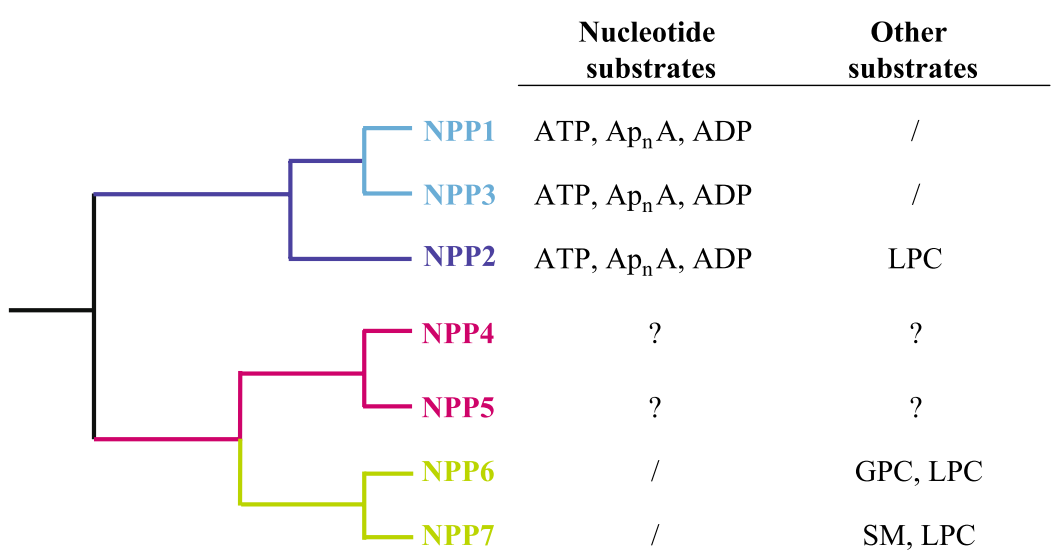

Figure 2. The phylogenetic tree of the NPP-family and some key substrates. Protein sequences for the human isoforms were retrieved from Genbank and aligned with CLUSTAL W. Accession numbers are: hNPP1, P22413; hNPP2, NP_006200.2; hNPP3, NP_005012.1; hNPP4, AAH18054.1; hNPP5, CAB56566.1; hNPP6, NP_699174.1; hNPP7, AAH41453.2. Representative nucleotide and/or lipid substrates are shown for each NPP isozyme. NPP1-3 have a common ancestor, and are the only known NPPs capable of hydrolyzing nucleotides. The overall aminoacid identity for the human isoenzymes, as obtained by Blast-2 sequence analysis at the NCBI site, is 41\% (NPP1-NPP2), 52\% (NPP1-NPP3) and 40\% (NPP2-NPP3). LPC Lysophosphatidylcholine, $G P C$ glycerophosporylcholine, $S M$ sphingomyelin. 
have been predicted to adopt a type-I orientation, with their $\mathrm{N}$-terminus facing the extracellular milieu [18, 20-22] (Figure 3). This prediction is supported experimentally for NPP6 and NPP7. Thus, truncated forms of NPP6 or NPP7 lacking the C-terminal putative transmembrane domain are not retained at the plasma membrane and are secreted $[22,25]$. In addition, a soluble form of NPP7 is released from the plasma membrane by trypsin, which acts on tryptic arginine site(s) located upstream of the transmembrane domain. Soluble forms have also been identified for NPP1, NPP3 and NPP6, but the mechanisms for their generation remains to be elucidated [22, 26-29]. NPP2 is synthesized as a pre-pro-enzyme and only exists as a secreted protein [30].

Our understanding of the structural and functional diversity of NPPs has increased considerably in the last few years. This review deals primarily with the domain structure and enzymatic properties of NPPs, and with their putative role in the modulation of purinergic signaling, in particular in relation to epithelial and neural functions, the immune response and cell motility. The reader can find complementary information on NPPs in other recent reviews $[31,32]$.

\section{Domain structure and catalytic properties of NPPs}

NPPs are modular proteins (Figure 3). In addition to a catalytic domain they also contain subcellular targeting or anchoring domains as well as regulatory domains.

\section{The catalytic domain: The NPP signature}

All NPPs have a catalytic domain of about 400 residues that shows up to $60 \%$ identity at the amino acid level between the different human isoforms. This catalytic domain is predicted to adopt a fold similar to that of alkaline phosphatases, phosphopentomutases and cofactorindependent phosphoglycerate mutases, which all belong to the superfamily of phospho-/sulfo-coordinating metalloenzymes [33, 34]. Also, the residues that coordinate two metals in the catalytic site of alkaline phosphatases and their spatial arrangement towards the catalytic site, are conserved in NPPs. Furthermore, the reaction mechanism of NPPs appears to be similar to that of the other phospho-/ sulfo-coordinating metalloenzymes and involves a two-step mechanism [18]. In the first step, the catalytic-site threonine/serine forms an intermediate with a phosphate group

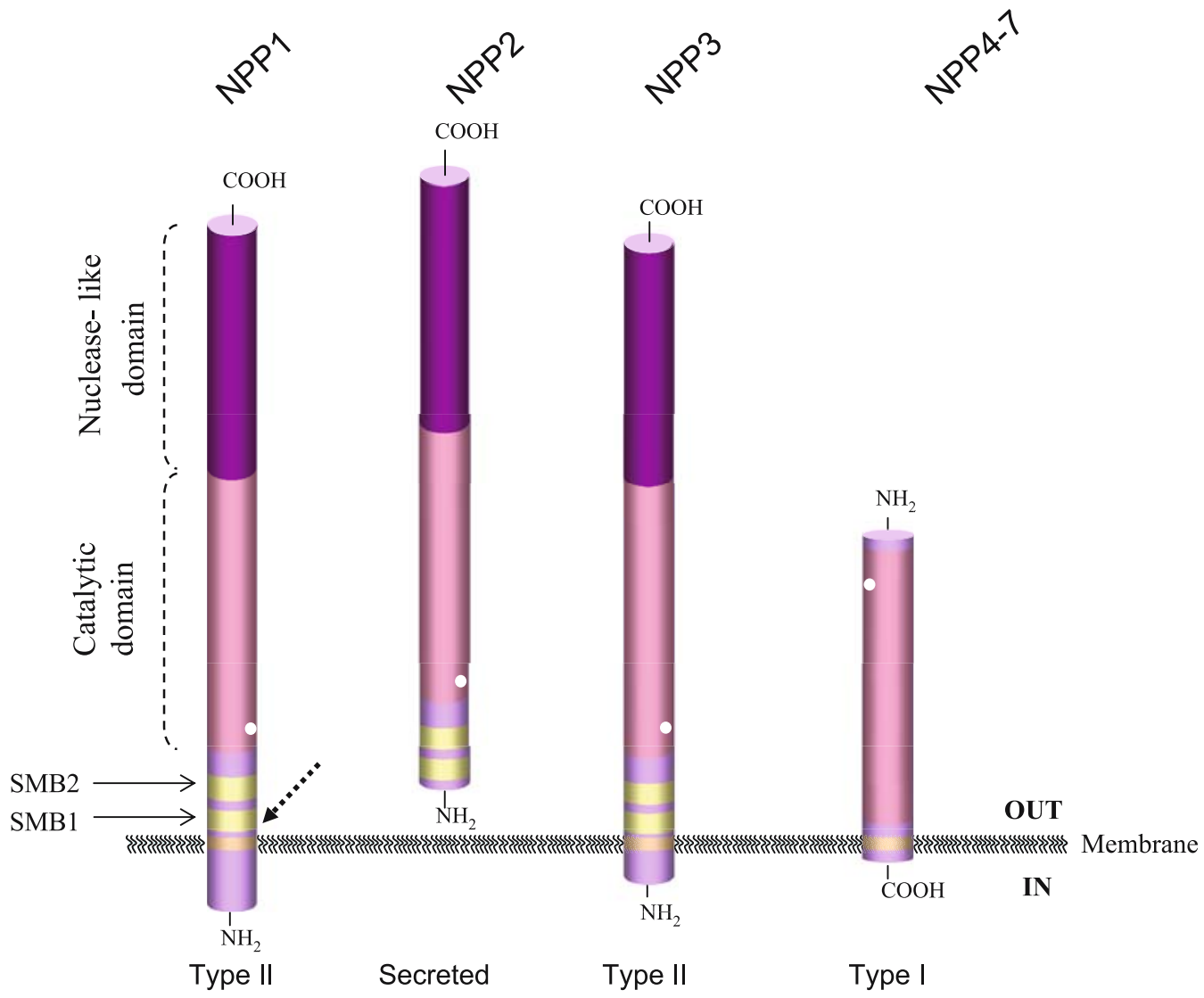

Figure 3. Domain structure and subcellular localisation of NPPs. Except for NPP2, which is secreted in the extracellular medium, NPP ectoenzymes are single-span membrane proteins with type-II (NPP1 and NPP3) or type-I orientation (NPP4-7). In all cases, the bulk of the protein lies outside the cell, with only short fragments facing the cytosol. Soluble NPP1 can be generated by cleavage of the membrane-associated form (arrow). The intracellular domains of NPP1 and NPP3 contain determinants for targeting to the basolateral or apical side of the plasma membrane, respectively. The common structural element of NPPs is the catalytic domain. Aminoacid identity of the catalytic domain, as obtained by Blast-2 sequence analysis of the human isoenzymes at the NCBI site is between 24\% (NPP2-NPP6) and 60\% (NPP1-NPP3). The position of the threonine/serine that mediates the formation of the catalytic intermediate is marked with white circles. The nuclease-like domain and the two somatomedin B-like domains (SMB1 and SMB2) are found only in NPP1-3. 
of the incoming substrate. In the second catalytic step, a water molecule is used to regenerate the catalytic-site threonine/serine and to release the phosphorylated product. For example, ATP is hydrolyzed into either AMP $+\mathrm{PP}_{\mathrm{i}}$ or $\mathrm{ADP}+\mathrm{P}_{\mathrm{i}}$, via an AMP- or phosphate-bound intermediate, respectively, depending on how the substrate approaches the catalytic site [18].

\section{The N-terminus: Signal peptide or signal anchor?}

The N-terminal 21-76 residues harbour the determinants for the subcellular localization of human NPPs. In NPP1 and NPP3 this region functions as a signal anchor, ensuring a type-II orientation across the membrane, with the Nterminus facing the cytosol. In the polarized epithelial cells, NPP1 and NPP3 are expressed at different sides of the plasma membrane. A di-leucine motif in the intracellular domain targets NPP1 to the basolateral membrane [35]. The N-terminus of NPP3 does not contain this motif and is therefore targeted apically, in part after it transiently reaches the basolateral area [36]. The first 27 residues of NPP2 function as a signal peptide that is removed cotranslationally by the signal peptidase in the endoplasmic reticulum. The resulting soluble pro-NPP2 is further cleaved by furin-type proteases along the secretory pathway to generate the mature, fully active NPP2 [30]. The N-terminus of NPP6-7 comprising about 20 residues functions as a signal peptide that is cleaved co-translationally. However, NPP6-7 have a C-terminal transmembrane domain, which accounts for their type-I transmembrane orientation, leaving the bulk of the protein extracellularly.

The somatomedin-B like domains of NPP1-3: An elusive function

Two cysteine-rich somatomedin-B like domains lie between the transmembrane and the catalytic domain of NPP1-3. One suggested function has been that of a 'stable stalk' between the transmembrane and the catalytic domains [10]. Also, NPP1 is a disulfide-linked homodimer and cysteines in these domains have been proposed to participate in interchain S-S bridges [18, 37]. However, the crystal structure of the somatomedin-B domain of vitronectin revealed that this domain forms a 'disulfide-knot', with all eight cysteines engaged in intrachain disulphide bonds [38]. This strongly indicates that the determinants for the dimerization of NPP1 lie outside the somatomedinB like domains. Consistent with this view, secreted NPP2 does not form dimers although it also has two somatomedin-B like domains [30]. It is therefore likely that in NPP1-3 these domains function as protein interaction domains, similarly to those in vitronectin [38].

\section{The C-terminal portion of NPP1-3: A multifunctional, nuclease-like domain}

A stretch of about 250 residues that flanks the catalytic domain of NPP1-3 C-terminally shows similarities with DNA/RNA non-specific endoribonucleases, both with respect to its primary structure and the predicted fold [37]. This 'nuclease-like' domain does not endow NPPs with a nuclease activity because the residues that are essential for catalysis by endoribonucleases are not conserved in NPP1-3. However, the nuclease-like domain does appear to contain isoform-specific determinants for catalysis by NPP1 and NPP2, since the swapping of their nuclease-like domain results in an inactive NPP2, while NPP1 with the nuclease-like domain of NPP2 is fully active [39]. The nuclease-like domain also appears to be essential for the translocation of NPPs from the endoplasmic reticulum to the Golgi-apparatus, possibly because it is required for the correct folding of NPPs [37]. Finally, recent evidence indicates that the nuclease-like domain of NPP2 has an anti-adhesion function and may function as a ligand for a G-protein coupled receptor [40].

\section{The substrate specificity of NPPs: Many unanswered questions}

NPPs can hydrolyze pyrophosphate or phosphodiester bonds in a wide variety of substrates, although each isoform has a well-defined substrate specificity (Figure 1). For example, NTPs and diadenosine polyphosphates are substrates for NPP1-3, while lysophosphatidylcholine is only hydrolyzed by NPP2 and NPP6. Remarkably, NPP2 and NPP6 hydrolyze distinct phosphodiester bonds in lysophosphatidylcholine, generating choline and phosphocholine, respectively. The determinants of the substrate specificity of NPPs are poorly understood. Clearly, the pyrophosphate or phosphodiester bond is not the only substrate-specifying element of NPPs. Site-directed mutagenesis and domain swapping studies revealed that the non-catalytic domains as well as residues near the catalytic site control the activity and substrate specificity of NPP1 and NPP2 [39]. The catalytic domain of NPP1 contains a GxGxxG motif that resembles part of a consensus dinucleotide-binding motif and is essential for catalysis by NPP1, but it does not appear to be a substrate-specifying motif [39]. Lipid-consensus motifs, similar to those found in phospholipases D are not present in NPP2. [41]. Nevertheless, it cannot be excluded that NPPs contain hitherto unrecognized nucleotide or lipid binding sites similarly to, for example, the substrate-specifying 'pockets' of serine proteases.

\section{Role of NPP1-3 in the metabolism of extracellular nucleotides}

NPP1-3, alone or in combination, are expressed in every cell type that has been analyzed for their presence [18, 31]. It therefore seems likely that these NPPs fulfill a broad range of functions. NPP isozymes may even 'moonlight,' in that they can fulfill multiple, apparently unrelated functions. For example, NPP1 is not only implicated in nucleotide metabolism but also appears to have an antiinsulin action by a mechanism that does not require catalytic activity [42, 43]. By far the best studied NPP 
function concerns the role of NPP1 in bone and soft-tissue mineralization $[44,45]$, which is the subject of a separate review in this issue. We only discuss here how NPP1-3 contribute, as nucleotide-metabolizing enzymes, to the epithelial and neural functions, the immune response and cell motility.

\section{NPP1-3 and epithelial functions}

Most epithelial cells release nucleotides, either constitutively or when challenged with stimuli like mechanical stress or hypotonicity-induced swelling. In the extracellular environment of epithelial cells, purinergic signaling is of prime importance for ion transport, cell-cell communication and cell migration [46-48].

\section{Airway epithelia}

Nucleotide metabolism at the surface of human airways has received much attention because of the critical role of nucleotides in the protective mechanism against bacterial and viral infections, known as mucociliary clearance (MCC) [49-53]. This defense mechanism involves complex signaling by diadenosine polyphosphates, ATP and adenosine, affecting overall fluid homeostasis via a control of epithelial chloride secretion and sodium uptake. In fact, nucleotide-based treatments combined with ecto-nucleotidase inhibitors are used for the improvement of MCC in chronic obstructive lung diseases such as cystic fibrosis.

Some efforts have been undertaken to identify the ectonucleotidases expressed by airway epithelia. There is good biochemical evidence for the presence of both NTPDase-type [51] and NPP-type activities [54] in the airway epithelia. Transcript analysis revealed the presence of various ectonucleotidases including NTPDase2, NTPDase3, NTPDase5 as well as NPP1-3 [55]. However, not all of them may be expressed at the protein level equally well. Importantly, human airway ectonucleotidases are cell-associated and are predominantly apical [51]. Within the NPP family, this suggests a role for NPP3, which is indeed targeted to the apical surface, while NPP1 is expressed at the basolateral one, and NPP2 only exists as a secreted form. However, NPP2 might also fulfill a critical function in the lung because the transcript level in the lung is among the highest of all tested organs [56]. In airways an NPP-type pyrophosphatase activity may be mainly involved in regulation of signaling by diadenosine polyphosphates, which were recently shown to bind to P2Y purinergic receptors distinct from those activated by ATP [57]. Other ectonucleotidases identified in human airway epithelia are the tissue non-specific alkaline phosphatase, exclusively expressed at the apical surface, and $5^{\prime}$ nucleotidases, expressed at both apical and basolateral sides [58].

\section{Liver epithelia}

The liver epithelial cells, i.e. hepatocytes and cholangiocytes, contribute to bile formation and secretion, one of the primary functions of the liver. Bile is formed by the parenchymal hepatocytes and is further enriched in bicarbonate by cholangiocytes, the cells that line the bile ducts [59, 60]. The biliary epithelium constitutively releases ATP into the bile from a vesicular storage site, and increase in cell volume promotes the exocytosis of ATP through a phosphoinositide 3-kinase-dependent mechanism [61]. Hepatocytes, too, contribute to the biliary pool of ATP [59]. NPP3 is the major NPP isoenzyme at the apical membrane of both hepatocytes and cholangiocytes $[62,63]$. An apical localization in hepatocytes has also been established for $5^{\prime}$-nucleotidase [59]. By regulating the breakdown of biliary ATP these nucleotide-hydrolyzing enzymes may interfere with purinergic signaling at the bile canaliculi, and thereby modulate the process of bile formation. In cholangiocytes, NPP3 may be one of the major apical ectonucleotidases, consistent with the functional expression of a single, dominant ATP degradation pathway at this site [64]. By contrast, NTPDase2/CD39L1 has been identified in portal fibroblasts and appears to be the main ectonucleotidase that indirectly controls purinergic signaling at the basolateral membrane of cholangiocytes [65]. The role of basolateral nucleotides in bile ductular secretion is relatively minor when compared to that of apical nucleotides, but there is evidence that basolateral nucleotides modulate the growth and differentiation of cholangiocytes [66].

The role of NPP3 in cholangiocytes may extend beyond the regulation of bile formation. Indeed, large amounts of NPP3 are found in the extracellular matrix and in the serum of chemically induced rat cholangiocarcinoma [67], an adenocarcinoma of intrahepatic bile ducts. Neoplastic transformation of biliary cells leads to an overexpression of NPP3 but also to its inappropriate targeting to the basolateral rather than to the apical side of the cholangiocytes. Since NPP3 can stimulate migration of cultured cells it may also be involved in metastasis of neoplastic cholangiocytes, by an as yet unknown mechanism [63].

NPP1 is also abundantly expressed in hepatocytes and localizes basolaterally [63]. An enticing hypothesis is that this pool of NPP1 controls the turnover of extracellular ATP, which functions as a potent hepatic mitogen by increasing immediate early gene expression in a P2receptor dependent manner [68]. Consistent with this notion, the level of NPP1 is considerably decreased during liver regeneration following 70\% partial hepatectomy and only reaches its initial level again after the major growth phase of the liver has passed [69]. We speculate that the transient drop in the concentration of NPP1 enables the accumulation of extracellular ATP, needed to stimulate cell proliferation.

\section{Intestinal epithelia}

Enterocytes constitute the major fraction of intestinal epithelial cells and their primary function is to absorb nutrients from the diet. Nucleotides are semi-essential or conditionally essential nutrients [70]. Cells can synthesize nucleotides de novo and re-use them through the nucleotide salvage pathway. However, rapidly proliferating tissues such as the intestine have an insufficient biosynthetic capacity and are dependent on an exogenous supply 
of nucleotides. NPP3 is the major NPP isoenzyme at the brush border of enterocytes [62]. Given its very high expression, combined with its co-localization with $5^{\prime}$ nucleotidase [71] or alkaline phosphatase, it seems likely that these ectonucleotidases play an important role in the digestion of dietary nucleotides and their derivatives but a direct evidence for this contention is lacking.

\section{NPP1-3 and neural functions}

Extracellular nucleotides, in particular ATP, have multiple functions in the central nervous system. ATP acts as a neurotransmitter and modulator of neurotransmitter release, but also as a trophic factor that stimulates proliferation and differentiation of neural cells [5, 72-75]. Neurons, glial cells and endothelial cells are established sources of extracellular purines. Initial evidence reveals important functions for NPPs in the nervous system, at least in part by their ability to modulate purinergic signaling.

Within the brain, a panel of $\mathrm{P} 2 \mathrm{X}$ receptors as well as NPP2 and NPP3 are expressed by the choroid-plexus epithelial cells [76-78]. The latter cells secrete cerebrospinal fluid (CSF), the major extracellular fluid in the central nervous system. CSF provides the central nervous system with nutrients and signaling molecules, and removes metabolites [79]. It seems likely that NPP $2 / 3$ contribute to the secretion of CSF by modulating purinergic signaling [78]. In rodents NPP2 can also be detected in the CSF itself. This pool of NPP2 possibly originates from the choroid-plexus epithelial cells and/or from the leptomeningeal cells and has recently been shown to function as a neurite retraction factor [80]. Remarkably, in humans
NPP2 is absent from the CSF of healthy persons, but is found in the CSF from patients with multiple sclerosis [81].

NPP2 is also secreted by oligodendrocytes, the myelin producing cells [82]. A tight correlation was noted between the expression of NPP2 in oligodendrocytes and the initial stages of myelination, a complex process that includes the movement of oligodendrocytes to the sites of myelination. NPP2 has been identified as an autocrine 'counteradhesive' factor and may thus be implicated in the movement of oligodendrocytes to their target sites [82]. Surprisingly, this function does not require the catalytic domain of NPP2 and appears to be mediated by the nuclease-like domain, which possibly interacts with a G-protein coupled receptor [40].

In the developing brain NPP3 is first scattered throughout the neuroepithelium and adjacent mesenchymal tissue, but later appears in distinct brain regions to reach the strongest expression on meninges, ependymal layers and choroid plexus [77]. The latter structures maintain a strong expression of NPP3 until adulthood. NPP3 was also found to be transiently expressed in a population of neural progenitor cells that can differentiate into radial glial-like cells, a subset of astrocytes and ependymal cells [77]. Since NPP3 is not expressed by mature astrocytes, this may suggest an involvement of this isoenzyme in keeping their progenitor cells dedifferentiated. Consistent with this notion, it was reported that the expression of NPP3 in NPP3-negative cell lines induces the expression of glial fibrillary acidic protein, an intermediate-filament protein specific for the cytoskeleton of astroglial cells and nonmyelin-forming Schwann cells. Interestingly, the overexpression of NPP3 has also been associated with increased motility and invasive properties of cultured cells [83].

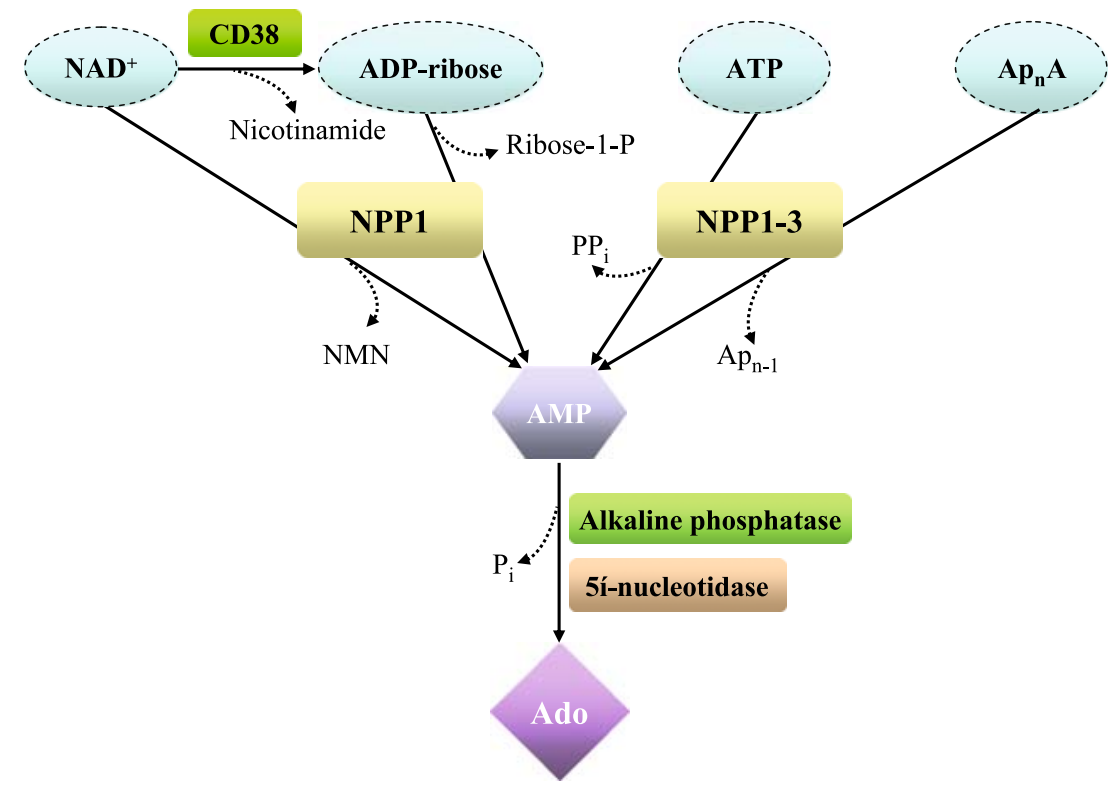

Figure 4. Role of NPP1-3 in the metabolism of extracellular nucleotides. NPP1 and CD38 are co-expressed in T-lymphocytes. Extracellular NAD ${ }^{+}$is a substrate for NPP1, a NAD ${ }^{+}$-pyrophosphatase, as well as for CD38, a NAD ${ }^{+}$-glycohydrolase. The coordinated expression of NPP1 and CD38 is part of a protective mechanism against $\mathrm{NAD}^{+}$-induced apoptosis of T cells. The hydrolysis of $\mathrm{NAD}^{+}$by the concerted action of NPP1, CD38 and $5^{\prime}$-nucleotidase also allows activated T cells to re-use the products for their own anabolic processes. At the apical membrane of hepatocytes and cholangiocytes, NPP3 hydrolyzes bile ATP and modulates purinergic signaling at these sites. Diadenosine polyphosphates $\left(\mathrm{Ap}_{3-5} \mathrm{~A}\right)$ and other nucleotides are hydrolyzed at the apical surface of the human airways by an NPP-type pyrophosphatase. Likely candidates are NPP3 and/or NPP2. 
Expression of NPP1 in glia cells under physiological conditions has not been reported. However, NPP1 is the major ectonucleotidase in the rat C6 glioma cell line [84, 85]. This cell line is morphologically similar to glioblastoma multiforme, the most common aggressive glioma resistant to therapeutic interventions [86].

\section{NPP1 and lymphocyte signaling}

NPP1 is a marker of a late stage in the differentiation of antibody-producing $\mathrm{B}$ cells, but its expression is not required for the synthesis and secretion of antibodies. Although initially believed to be lineage-specific [87], in the meantime it has been established that low levels of NPP1 are also found in T cells and that its expression in these cells is increased through signaling by protein kinases $\mathrm{A}$ and $\mathrm{C}$, the same pathways that also up-regulate the expression of the $\mathrm{NAD}^{+}$glycohydrolase CD38 [88]. The coordinated expression of NPP1 and CD38 enables the hydrolysis of extracellular $\mathrm{NAD}^{+}$by activated $\mathrm{T}$ cells to nicotinamide mononucleotide + AMP or to nicotinamide + ADP-ribose, respectively (Figure 4). In doing so, NPP1 and $\mathrm{CD} 38$ control the substrate availability for $\mathrm{NAD}^{+}$-dependent ADP-ribosylation by ADP-ribosyltransferases at the cell surface. This can be seen as a protective mechanism against apoptosis at sites of inflammation, where the release of $\mathrm{NAD}^{+}$results in ADP-ribosylation and activation of the pro-apoptotic $\mathrm{P} 2 \mathrm{X}_{7}$ receptors $[89,90]$. By the same mechanism NPP1 and CD38 also provide a protection against the ADP-ribosylating bacterial toxins, such as cholera or C. botulinum $\mathrm{C} 3$ toxins, which exert their cytotoxic effects through ADP-ribosylation of host signaling molecules [91]. Both $\mathrm{NAD}^{+}$and its degradation product ADP-ribose are potent inhibitors of T-cell proliferation [92]. Their hydrolysis by CD38 and NPP1 is thus expected to promote $\mathrm{T}$-cell proliferation. Finally, in combination with $5^{\prime}$ nucleotidase, CD38 and NPP1 are part of the nucleotide-salvage pathway [88] (Figure 4).

\section{NPP2 and cell motility}

NPP2 is secreted by various cancer cells including skin, lung and breast cancer cells. Its tumour growth and motility effects have been primarily attributed to its ability to produce lysophosphatidic acid (LPA) from lysophosphatidylcholine (LPC) [93]. LPA binds to dedicated Gprotein-coupled receptors, $\mathrm{LPA}_{1-4}$, and activates multiple signaling pathways, leading to cell proliferation, cell-shape changes and migration. These effects explain why NPP2 promotes tumour growth, angiogenesis and metastasis (Figure 5). However, it can be envisaged that NPP2 also promotes tumour growth by its ability to hydrolyze nucleotides. Indeed, solid tumours are well known to release adenine nucleotides and NPP2 can hydrolyze ATP, which is an inhibitor of tumour-cell proliferation [49]. Moreover, the concerted action of NPP2 and $5^{\prime}$-nucleotidase on ATP and ADP generates adenosine, a tumour-growth promoter and stimulator of angiogenesis (Figure 4) [94-96]. In this respect, it is also worthy of note

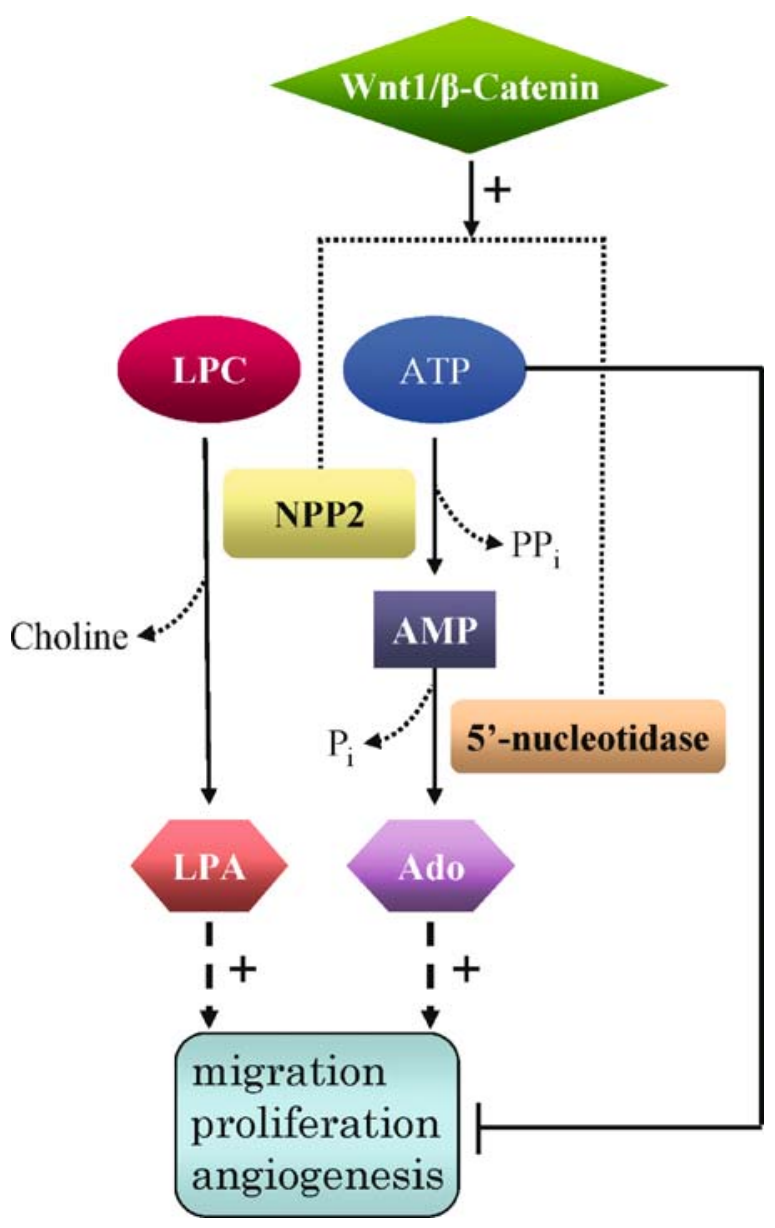

Figure 5. Tumour growth and metastasis: Role of NPP2-catalyzed reactions. In the extracellular milieu the tumour-motility stimulating factor NPP2 generates LPA from LPC. LPA promotes cell proliferation, migration and angiogenesis. In a coupled reaction with $5^{\prime}$-nucleotidase, NPP2 has the potential to hydrolyze ATP, which is cytotoxic for tumours, to adenosine, a tumour-growth promoting factor and stimulator of angiogenesis. The expression of both NPP2 and $5^{\prime}$-nucleotidase is increased by the Wnt/ $\beta$-catenin pathway, which is activated in many cancers.

that the $W n t / \beta$-catenin signaling pathway is activated in many human tumours, resulting in an increased expression of both NPP2 [97, 98] and 5'-nucleotidase [99].

\section{Conclusions and perspectives}

The family of NPP-type ectophosphodiesterases comprises a versatile group of seven ecto-enzymes that hydrolyze pyrophosphate and phosphodiester bonds in a broad range of metabolites, yet each isozyme has a rather narrow substrate-specificity. Only NPP1-3 are known to hydrolyze nucleotides and they are part of a complex nucleotide interconversion network at the cell surface. NPP1-3 contribute to ectonucleotide signaling both by removing active compounds and by generating nucleotide metabolites with distinct signaling properties. The complexity of signaling via NPPs is further increased by their ability to hydrolyze non-nucleotide substrates. For example, NPP2 can hydrolyze both phospholipids and nucleo- 
tides and the generated products have the potential of acting antagonistically or synergistically. A surprising finding has been that NPP1 and NPP2 have also functions that do not require catalytic activity. This can imply that NPPs moonlight, although it cannot be ruled out that their noncatalytic functions are somehow connected to their catalytic functions.

Much remains to be learned about the enzymatic properties, regulation and functional diversity of NPPs. A lot is expected from the phenotyping of knockout models for each NPP isoenzyme. Also, the availability of isoenzyme-specific NPP inhibitors would be of great help to study their function. The latter also have therapeutic potential as they hold great promises for the treatment of devastating diseases like cystic fibrosis and bone-mineralization pathologies.

\section{Acknowledgments}

The authors' work on NPPs is supported by the Fund for Scientific Research - Flanders (grant G.0082.02). Silvia Jansen is a Research Assistant of the Fund for Scientific Research.

\section{References}

1. Boeynaems JM, Communi D, Gonzalez NS, Robaye B. Overview of the P2 receptors. Semin Thromb Hemost 2005; 31: 139-49.

2. Burnstock G. Purinergic signaling and vascular cell proliferation and death. Arterioscler Thromb Vasc Biol 2002; 22: 364-73.

3. Burnstock G. Potential therapeutic targets in the rapidly expanding field of purinergic signaling. Clin Med 2002; 2: 45-53.

4. Sak K, Boeynaems JM, Everaus H. Involvement of P2Y receptors in the differentiation of haematopoietic cells. J Leukoc Biol 2003; 73: 442-7.

5. Agresti $\mathrm{C}$, Meomartini ME, Amadio $\mathrm{S}$ et al. ATP regulates oligodendrocyte progenitor migration, proliferation, and differentiation: Involvement of metabotropic P2 receptors. Brain Res Rev 2005; 48: 157-65.

6. Lazarowski ER, Boucher RC, Harden TK. Mechanisms of release of nucleotides and integration of their action as P2X- and P2Y-receptor activating molecules. Mol Pharmacol 2003; 64: 785-95.

7. Yegutkin GG, Henttinen T, Jalkanen S. Extracellular ATP formation on vascular endothelial cells is mediated by ecto-nucleotide kinase activities via phosphotransfer reactions. FASEB J 2001; 15: 251-60.

8. Donaldson SH, Picher M, Boucher RC. Secreted and cell-associated adenylate kinase and nucleoside diphosphokinase contribute to extracellular nucleotide metabolism on human airway surfaces. Am J Respir Cell Mol Biol 2002; 26: 209-15.

9. Picher M, Boucher RC. Human airway ecto-adenylate kinase. A mechanism to propagate ATP signaling on airway surfaces. J Biol Chem 2003; 278: 11256-64.

10. Zimmermann H. Extracellular metabolism of ATP and other nucleotides. Naunyn-Schmiedebergs Arch Pharmacol 2000; 362: 299-309.

11. Kunapuli SP, Dorsam RT, Kim S, Quinton TM. Platelet purinergic receptors. Curr Opin Pharmacol 2003; 3: 175-80.

12. Dorsam RT, Kunapuli SP. Central role of the P2Y12 receptor in platelet activation. J Clin Invest 2004; 113: 340-5.

13. Sitkovsky M, Lukashev D. Regulation of immune cells by localtissue oxygen tension: HIF1 alpha and adenosine receptors. Nat Rev Immunol 2005; 5: 712-21.
14. Hershfield MS. New insights into adenosine-receptor-mediated immunosuppression and the role of adenosine in causing the immunodeficiency associated with adenosine deaminase deficiency. Eur J Immunol 2005; 35: 25-30.

15. Adair TH. Growth regulation of the vascular system: An emerging role for adenosine. Am J Physiol Regul Integr Comp Physiol 2005; 289: R283-96.

16. Sevigny J, Sundberg C, Braun N et al. Differential catalytic properties and vascular topography of murine nucleoside triphosphate diphosphohydrolase 1 (NTPDase1) and NTPDase2 have implications for thromboregulation. Blood 2002; 99: 2801-9.

17. Heine P, Braun N, Heilbronn A et al. Functional characterization of rat ecto-ATPase and ecto-ATP diphosphohydrolase after heterologous expression in CHO cells. Eur J Biochem 1999; 262:102-7.

18. Bollen M, Gijsbers R, Ceulemans H, Stefan C. Nucleotide pyrophosphatase/phosphodiesterases on the move. Crit Rev Biochem Mol Biol 2000; 35: 393-432.

19. Vollmayer P, Clair T, Goding JW et al. Hydrolysis of diadenosine polyphosphates by nucleotide pyrophosphatases/phosphodiesterases. Eur J Biochem 2003; 270: 2971-8.

20. Duan RD, Bergman $\mathrm{T}, \mathrm{Xu} \mathrm{N}$ et al. Identification of human intestinal alkaline sphingomyelinase as a novel ecto-enzyme related to the nucleotide phosphodiesterase family. J Biol Chem 2003; 278: 38528-36.

21. Wu J, Cheng Y, Palmberg $\mathrm{C}$ et al. Cloning of alkaline sphingomyelinase from rat intestinal mucosa and adjusting of the hypothetical protein XP_221184 in GenBank. Biochim Biophys Acta 2005; 1687: 94-102.

22. Sakagami H, Aoki J, Natori Y et al. Biochemical and molecular characterization of a novel choline-specific glycerophosphodiester phosphodiesterase belonging to the nucleotide pyrophosphatase/ phosphodiesterase family. J Biol Chem 2005; 280: 23084-93.

23. Umezu-Goto M, Kishi Y, Taira A et al. Autotaxin has lysophospholipase D activity leading to tumour cell growth and motility by lysophosphatidic acid production. J Cell Biol 2002; 158: 227-33.

24. Tokumura A, Majima E, Kariya $\mathrm{Y}$ et al. Identification of human plasma lysophospholipase D, a lysophosphatidic acid-producing enzyme, as autotaxin, a multifunctional phosphodiesterase. J Biol Chem 2002; 277: 39436-42.

25. Wu J, Liu F, Nilsson A et al. Pancreatic trypsin cleaves intestinal alkaline sphingomyelinase from mucosa and enhances the sphingomyelinase activity. Am J Physiol Gasterointest Liver Physiol 2004; 287:G967-73.

26. Belli SI, van Driel IR, Goding JW. Identification and characterization of a soluble form of the plasma cell membrane glycoprotein PC1 (5'-nucleotide phosphodiesterase). Eur J Biochem 1993; 217: $421-8$.

27. Hosoda N, Hoshino SI, Kanda Y, Katada T. Inhibition of phosphodiesterase/pyrophosphatase activity of PC-1 by its association with glycosaminoglycans. Eur J Biochem 1999; 265: 763-70.

28. Meerson NR, Delautier D, Durand-Schneider AM et al. Identification of B10, an alkaline phosphodiesterase of the apical plasma membrane of hepatocytes and biliary cells, in rat serum: Increased levels following bile duct ligation and during the development of cholangiocarcinoma. Hepatology 1998; 27: 563-8.

29. Yegutkin GG, Samburski SS, Jalkanen S. Soluble purine-converting enzymes circulate in human blood and regulate extracellular ATP level via counteracting pyrophosphatase and phosphotransfer reactions. FASEB J 2003; 17: 1328-30.

30. Jansen S, Stefan C, Creemers JW et al. Proteolytic maturation and activation of autotaxin (NPP2), a secreted metastasis-enhancing lysophospholipase D. J Cell Sci 2005; 118: 3081-9.

31. Goding JW, Grobben B, Slegers H. Physiological and pathophysiological functions of the ecto-nucleotide pyrophosphatase/phosphodiesterase family. Biochim Biophys Acta 2003; 1638: 1-19.

32. Stefan C, Jansen S, Bollen M. NPP-type ectophosphodiesterases: Unity in diversity. Trends Biochem Sci 2005; 30: 542-50.

33. Galperin MY, Bairoch A, Koonin EV. A superfamily of metalloenzymes unifies phosphopentomutase and cofactor-independent 
phosphoglycerate mutase with alkaline phosphatases and sulfatases. Prot Sci 1998; 7: 1829-35.

34. Gijsbers R, Ceulemans H, Stalmans W, Bollen M. Structural and catalytic similarities between nucleotide pyrophosphatases/phosphodiesterases and alkaline phosphatases. J Biol Chem 2001; 276: $1361-8$.

35. Vaingankar SM, Fitzpatrick TA, Johnson K et al. Subcellular targeting and function of osteoblast nucleotide pyrophosphatase phosphodiesterase 1. Am J Physiol Cell Physiol 2004; 286: C1177-87.

36. Meerson NR, Bello V, Delaunay JL et al. Intracellular traffic of the ecto-nucleotide pyrophosphatase/phosphodiesterase NPP3 to the apical plasma membrane of MDCK and Caco-2 cells: Apical targeting occurs in the absence of $N$-glycosylation. J Cell Sci 2000; 113: 4193-202.

37. Gijsbers R, Ceulemans H, Bollen M. Functional characterization of the non-catalytic ectodomains of the nucleotide pyrophosphatase/ phosphodiesterase NPP1. Biochem J 2003; 371: 321-30.

38. Zhou A, Huntington JA, Pannu NS et al. How vitronectin binds PAI1 to modulate fibrinolysis and cell migration. Nat Struct Biol 2003; 10: $541-4$.

39. Cimpean A, Stefan C, Gijsbers R et al. Substrate-specifying determinants of the nucleotide pyrophosphatases/phosphodiesterases NPP1 and NPP2. Biochem J 2004; 381: 71-7.

40. Fox MA, Alexander JK, Afshari FS et al. Phosphodiesterase-I alpha/ autotaxin controls cytoskeletal organization and FAK phosphorylation during myelination. Mol Cell Neurosci 2004; 27: 140-50.

41. Frohman MA, Sung TC, Morris AJ. Mammalian phospholipase D structure and regulation. Biochim Biophys Acta 1999; 1439: 175-86.

42. Grupe A, Alleman J, Goldfine ID et al. Inhibition of insulin receptor phosphorylation by PC-1 is not mediated by the hydrolysis of adenosine triphosphate or the generation of adenosine. J Biol Chem 1995; 270: 22085-8.

43. Stefan C, Wera S, Stalmans W, Bollen M. The inhibition of the insulin receptor by the receptor protein PC-1 is not specific and results from the hydrolysis of ATP. Diabetes 1996; 45: 980-3.

44. Hessle L, Johnson KA, Anderson $\mathrm{HC}$ et al. Tissue-nonspecific alkaline phosphatase and plasma cell membrane glycoprotein-1 are central antagonistic regulators of bone mineralization. Proc Natl Acad Sci USA 2002; 99: 9445-9.

45. Rutsch F, Ruf N, Vaingankar S et al. Mutations in ENPP1 are associated with 'idiopathic' infantile arterial calcification. Nat Genet 2003; 34: 379-81.

46. Bucheimer RE, Linden J. Purinergic regulation of epithelial transport. J Physiol 2004; 555: 311-21.

47. Schwiebert EM, Zsembery A. Extracellular ATP as a signaling molecule for epithelial cells. Biochim Biophys Acta 2003; 1615: $7-32$.

48. Klepeis VE, Weinger I, Kaczmarek E, Trinkaus-Randall V. P2Y receptors play a critical role in epithelial cell communication and migration. J Cell Biochem 2004; 93: 1115-33.

49. Agteresch HJ, Dagnelie PC, van den Berg JW, Wilson JH. Adenosine triphosphate: Established and potential clinical applications. Drugs 1999; 58: 211-32.

50. Kellerman DJ. P2Y(2) receptor agonists: A new class of medication targeted at improved mucociliary clearance. Chest 2002; 121: 201S-5S.

51. Picher M, Burch LH, Boucher RC. Metabolism of P2 receptor agonists in human airways: Implications for mucociliary clearance and cystic fibrosis. J Biol Chem 2004; 279: 20234-41.

52. Lazarowski ER, Tarran R, Grubb BR et al. Nucleotide release provides a mechanism for airway surface liquid homeostasis. J Biol Chem 2004; 279: 36855-64.

53. Taira M, Tamaoki J Nishimura $\mathrm{K}$ et al. Adenosine A(3) receptormediated potentiation of mucociliary transport and epithelial ciliary motility. Am J Physiol Lung Cell Mol Physiol 2002; 282: L556-62.

54. Picher M, Boucher RC. Biochemical evidence for an ectoalkaline phosphodiesteraseI in human airways. Am J Respir Cell Mol Biol 2000; 23: 255-61.
55. Picher M, Boucher RC. Metabolism of extracellular nucleotides in human airways by a multienzyme system. Drug Dev Res 2001; 52: 66-75.

56. Stefan C, Gijsbers R, Stalmans W, Bollen M. Differential regulation of the expression of nucleotide pyrophosphatases/phosphodiesterases in rat liver. Biochim Biophys Acta 1999; 1450: 45-52.

57. Laubinger $\mathrm{W}$, Wang $\mathrm{H}$, Welte $\mathrm{T}$ et al. $\mathrm{P} 2 \mathrm{Y}$ receptor specific for diadenosine tetraphosphate in lung: Selective inhibition by suramin, PPADS, Ip5I, and not by MRS-2197. Eur J Pharmacol 2003; 468: 9-14.

58. Picher M, Burch LH, Hirsh AJ et al. Ecto $5^{\prime}$-nucleotidase and nonspecific alkaline phosphatase. Two AMP-hydrolyzing ectoenzymes with distinct roles in human airways. J Biol Chem 2003; 278:13468-79.

59. Che M, Gatmaitan Z, Arias IM. Ectonucleotidases, purine nucleoside transporter, and function of the bile canalicular plasma membrane of the hepatocyte. FASEB J 1997; 11: 101-8.

60. Hirata $\mathrm{K}$, Nathanson MH. Bile duct epithelia regulate biliary bicarbonate excretion in normal rat liver. Gastroenterology 2001; 121: 396-406.

61. Gatof D, Kilic G, Fitz JG. Vesicular exocytosis contributes to volume-sensitive ATP release in biliary cells. Am J Physiol Gasterointest Liver Physiol 2004; 286: G538-46.

62. Scott LJ, Delautier D, Meerson NR et al. Biochemical and molecular identification of distinct forms of alkaline phosphodiesterase I expressed on the apical and basolateral plasma membrane surfaces of rat hepatocytes. Hepatology 1997; 25: 995-1002.

63. Yano Y, Hayashi Y, Sano K et al. Expression and localization of ecto-nucleotide pyrophosphatase/phosphodiesterase I-1 (E-NPP1/ PC-1) and -3 (E-NPP3/CD203c/PD-Ibeta/B10/gp130(RB13-6)) in inflammatory and neoplastic bile duct diseases. Cancer Lett 2004; 207: 139-147.

64. Salter KD, Fitz JG, Roman RM. Domain-specific purinergic signaling in polarized rat cholangiocytes. Am J Physiol Gasterointest Liver Physiol 2000; 278: G492-500.

65. Dranoff JA, Kruglov EA, Robson SC et al. The ecto-nucleoside triphosphate diphosphohydrolase NTPDase2/CD39L1 is expressed in a novel functional compartment within the liver. Hepatology 2002; 36: $1135-44$

66. Jhandier MN, Kruglov EA, Lavoie EG et al. Portal fibroblasts regulate the proliferation of bile duct epithelia via expression of NTPDase2. J Biol Chem 2005; 280: 22986-92.

67. Scoazec JY, Moreau A, Maurice M et al. Detection of a biliary cell membrane glycoprotein in the serum of cholangiocarcinoma-bearing rats. Possible relevance to the membrane proteins used as serum tumour markers in humans. Lab Invest 1990; 62: 459-66.

68. Thevananther S, Sun H, Li D, Arjunan V et al. Extracellular ATP activates c-Jun N-terminal kinase signaling and cell cycle progression in hepatocytes. Hepatology 2004; 39: 393-402.

69. Stefan C, Stalmans W, Bollen M. Growth-related expression of the ectonucleotide pyrophosphatase PC-1 in rat liver. Hepatology 1998; 28: $1497-503$.

70. Aggett $\mathrm{P}$, Leach JL, Rueda $\mathrm{R}$ et al. Innovation in infant formula development: A reassessment of ribonucleotides in 2002. Nutrition $2003 ; 19: 375-84$.

71. Synnestvedt K, Furuta GT, Comerford KM et al. Ecto-5'-nucleotidase (CD73) regulation by hypoxia-inducible factor-1 mediates permeability changes in intestinal epithelia. J Clin Invest 2002; 110: 993-1002.

72. Neary JT, Kang Y, Shi YF. Signaling from nucleotide receptors to protein kinase cascades in astrocytes. Neurochem Res 2004; 29: 2037-42.

73. Koizumi S, Fujishita K, Inoue K. Regulation of cell-to-cell communication mediated by astrocytic ATP in the CNS. Purinergic Signalling 2005; 1: 211-7.

74. Franke H, Illes P. Involvement of P2 receptors in the growth and survival of neurons in the CNS. Pharmacol Ther 2006; 109: 297-324.

75. Agresti C, Meomartini ME, Amadio S et al. Metabotropic P2 receptor activation regulates oligodendrocyte progenitor migration and development. Glia 2005; 50: 132-44. 
76. Fuss B, Baba H, Phan T et al. Phosphodiesterase I, a novel adhesion molecule and/or cytokine involved in oligodendrocyte function. J Neurosci 1997; 17: 9095-103.

77. Blass-Kampmann S, Kindler-Rohrborn A, Deissler $\mathrm{H}$ et al. In vitro differentiation of neural progenitor cells from prenatal rat brain: Common cell surface glycoprotein on three glial cell subsets. J Neurosci Res 1997; 48: 95-111.

78. Xiang Z, Burnstock G. Expression of P2X receptors in rat choroid plexus. Neuroreport 2005; 16: 903-07.

79. Brown PD, Davies SL, Speake T, Millar ID. Molecular mechanisms of cerebrospinal fluid production. Neuroscience 2004; 129: 957-70.

80. Sato K, Malchinkhuu E, Muraki T et al. Identification of autotaxin as a neurite retraction-inducing factor of $\mathrm{PC} 12$ cells in cerebrospinal fluid and its possible sources. J Neurochem 2005; 92: 904-14.

81. Hammack BN, Fung KY, Hunsucker SW et al. Proteomic analysis of multiple sclerosis cerebrospinal fluid. Mult Scler 2004; 10: 245-60.

82. Fox MA, Colello RJ, Macklin WB, Fuss B. Phosphodiesterase-Ialpha/ autotaxin: A counteradhesive protein expressed by oligodendrocytes during onset of myelination. Mol Cell Neurosci 2003; 23: 507-519.

83. Deissler H, Blass-Kampmann S, Bruyneel E et al. Neural cell surface differentiation antigen gp130(RB13-6) induces fibroblasts and glioma cells to express astroglial proteins and invasive properties. FASEB J 1999; 13: 657-66.

84. Grobben B, Anciaux K, Roymans D et al. An ecto-nucleotide pyrophosphatase is one of the main enzymes involved in the extracellular metabolism of ATP in rat C6 glioma. J Neurochem 1999; 72: 826-34.

85. Grobben B, Claes P, Roymans D et al. Ecto-nucleotide pyrophosphatase modulates the purinoceptor-mediated signal transduction and is inhibited by purinoceptor antagonists. Br J Pharmacol 2000; 130: $139-45$.

86. Grobben B, De Deyn PP, Slegers H. Rat C6 glioma as experimental model system for the study of glioblastoma growth and invasion. Cell Tissue Res 2002; 310: 257-270.

87. Anderson KC, Bates MP, Slaughenhoupt B et al. A monoclonal antibody with reactivity restricted to normal and neoplastic plasma cells. J Immunol 1984; 132: 3172-9.
88. Deterre $\mathrm{P}$, Gelman L, Gary-Gouy $\mathrm{H}$ et al. Coordinated regulation in human $\mathrm{T}$ cells of nucleotide-hydrolyzing ecto-enzymatic activities, including CD38 and PC-1. Possible role in the recycling of nicotinamide adenine dinucleotide metabolites. J Immunol 1996; 157: 1381-8.

89. Seman M, Adriouch S, Scheuplein F et al. NAD-induced T cell death: ADP-ribosylation of cell surface proteins by ART2 activates the cytolytic P2X7 purinoceptor. Immunity 2003; 19: 571-82.

90. Krebs C, Adriouch S, Braasch F et al. CD38 controls ADPribosyltransferase-2-catalyzed ADP-ribosylation of $\mathrm{T}$ cell surface proteins. J Immunol 2005; 174: 3298-305.

91. Bortell R, Moss J, McKenna RC et al. Nicotinamide adenine dinucleotide (NAD) and its metabolites inhibit $\mathrm{T}$ lymphocyte proliferation: Role of cell surface NAD glycohydrolase and pyrophosphatase activities. J Immunol 2001; 167: 2049-59.

92. Popoff MR. Interactions between bacterial toxins and intestinal cells. Toxicon 1998; 36: 665-85.

93. Moolenaar WH. Lysophospholipids in the limelight: Autotaxin takes center stage. J Cell Biol 2002; 158: 197-9.

94. Spychala J. Tumour-promoting functions of adenosine. Pharmacol Ther 2000; 87: 161-73.

95. Cronstein BN. Adenosine receptors and wound healing. Sci World J 2004; 4: 1-8.

96. Desai A, Victor-Vega C, Gadangi S et al. Adenosine A2A receptor stimulation increases angiogenesis by down-regulating production of the antiangiogenic matrix protein thrombospondin 1. Mol Pharmacol. 2005; 67: 1406-13.

97. Tice DA, Szeto W, Soloviev I et al. Synergistic induction of tumour antigens by Wnt-1 signaling and retinoic acid revealed by gene expression profiling. J Biol Chem 2002; 277: 14329-35.

98. Kenny PA, Enver T, Ashworth A. Receptor and secreted targets of Wnt-1/beta-catenin signaling in mouse mammary epithelial cells. BMC Cancer 2005; 5: 3-11.

99. Spychala J, Kitajewski J. Wnt and beta-catenin signaling target the expression of ecto-5'-nucleotidase and increase extracellular adenosine generation. Exp Cell Res 2004; 296: 99-108. 